\title{
Global patterns and determinants of lake macrophyte taxonomic, functional and phylogenetic beta diversity
}

Jorge García-Girón a, ?], Jani Heino ${ }^{b}$, Lars Baastrup-Spohr ${ }^{c}$, Claudia P. Bove ${ }^{d}$, John Clayton ${ }^{\text {, }}$, Mary de Winton e, Tõnu Feldmann ${ }^{f}$, Margarita Fernândez-Alâez a , Frauke Ecke g,h, Patrick Grillasi, Mark V. Hoyeri, Agnieszka Kolada k, Sarian Kosten', Balâzs A. Lukâcs m, Marit Mjelde n, Roger P. Mormul ${ }^{\circ}$, Laila Rhazi $p$, Mouhssine Rhazi ${ }^{\text {, Laura Sass }}{ }^{r}$, Jun Xu ${ }^{\text {s }}$, Janne Alahuhta b,t

a Ecology Unit, University of León, Campus de Vegazana S/N, 24071 León, Spain

${ }^{b}$ Finnish Environment Institute, Freshwater Centre, P.O. Box 413, 90014 Oulu, Finland

${ }^{c}$ Freshwater Biological Laboratory, Department of Biology, University of Copenhagen, Universitetsparken 4, 2100 København $\varnothing$,Denmark

d Departamento de Botânica, Museu Nacional, Universidade Federal do Rio de Janeiro, Quinta da Boa Vista, Rio de Janeiro, RJ 20940-040, Brazil

e National Institute of Water and Atmospheric Research Limited, P.O. Box 11115, Hamilton, New Zealand

f Centre for Limnology, Institute ofAgricultural and Environmental Sciences, Estonian University of Life Sciences, 61117 Rannu, Tartumaa, Estonia

$\mathrm{g}$ Department ofAquatic Sciences andAssessment, Swedish University ofAgricultural Sciences (SLU), P.O. Box 7050, 75007 Uppsala, Sweden

$\mathrm{h}$ Department ofWildlife, Fish and Environmental Studies, Swedish University ofAgricultural Sciences (SLU), 90183 Umeå, Sweden

i Tour du Valat, Research Institute for the Conservation of Mediterranean Wetlands, Le Sambuc, 13200 Arles, France

${ }^{j}$ Fisheries and Aquatic Sciences, School of Forest Resources and Conservation, Institute of Food and Agricultural Services, University of Florida, 7922 NW 71st Street, Gainesville, FL 32609 , USA

$\mathrm{k}$ Department of Freshwater Protection, Institute of Environmental Protection-National Research Institute, Krucza 5/11D, 00-548 Warsaw, Poland

I Department of Aquatic Ecology and Environmental Biology, Institute for Water and Wetland Research, Radboud University, Heyendaalseweg 135, 6525AJ Nijmegen, the Netherlands

$\mathrm{m}$ Department of Tisza River Research, MTA Centre for Ecological Research, DRI, Bem tér 18/C, Debrecen 4026, Hungary

${ }^{n}$ Norwegian Institute for Water Research (NIVA), Gaustadalléen 21, 0349 Oslo, Norway

o Department of Biology, Research Group in Limnology, Ichthyology and Aquaculture-Nupélia, State University of Maringá, Av. Colombo 5790, Bloco H90, CEP-87020-900 Mringá, PR, Brazil

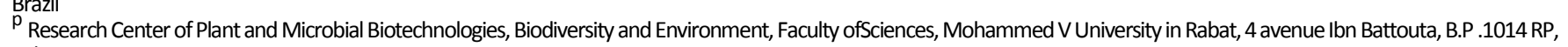
Rabat, Morocco

${ }^{q}$ Faculty of Science and Technology, Department of Biology, Moulay Ismail University, PB 509, Boutalamine, Errachidia, Morocco

${ }^{r}$ Illinois Natural History Survey, Prairie Research Institute, University of Illinois, 1816 South Oak Street, Champaign, IL 61820, USA

s Institute of Hydrobiology, Chinese Academy of Sciences, Wuhan 430070, China

${ }^{\mathrm{t}}$ Geography Research Unit, University of Oulu, P.O. Box 3000, 90014 Oulu, Finland

Corresponding author. :E-mail addresses: jogarg@unileon.es (J. García-Girón), jani.heino@environment.fi (J. Heino), lbaastrupspohr@bio.ku.dk (L. Baastrup-Spohr), John.Clayton@niwa.co.nz (J. Clayton), Mary.deWinton@niwa.co.nz (M. de Winton), Tonu.Feldmann@emu.ee (T. Feldmann), mfera@unileon.es (M. Fernândez-Alâez), frauke.ecke@slu.se (F. Ecke), grillas@tourduvalat.org (P.Grillas), mvhoyer@ufl.edu (M.V. Hoyer), agnieszka.kolada@ios.edu.pl (A. Kolada), s.kosten@science.ru.nl (S.' Kosten), lukacs.balazs@@okologia.mta.hu (B.A. Lukâcs), marit.mjelde@niva.no (M. Mjelde), Icanny@illinois.edu (L. Sass), xujun@ihb.ac.cn (J. Xu), Janne.Alahuhta@oulu.fi (J. Alahuhta).

How to cite : García-Girón J., Heino J., Baastrup-Spohr L., Bove C.P., Clayton J., de Winton M., Feldmann T., FernándezAláez M., Ecke F., Grillas P., Hoyer M.V., Kolada A., Kosten S., Lukács B.A., Mjelde M., Mormul R.P., Rhazi L., Rhazi M., Sass L., Xu J., Alahuhta J. 2020. Global patterns and determinants of lake macrophyte taxonomic, functional and phylogenetic beta diversity. Science of The Total Environment 723:138021. doi: 10.1016/i.scitotenv.2020.138021

\section{Abstract}

Documenting the patterns of biological diversity on Earth has always been a central challenge in macroecology and biogeography. However, for the diverse group of freshwater plants, such research program is still in its in- fancy. Here, we examined global variation in taxonomic, functional and phylogenetic beta diversity patterns of lake macrophytes using regional data from six continents. A data set of ca. 480 lake macrophyte community observations, together with climatic, geographical and environmental variables, was compiled across 16 regions worldwide. We (a) built the very first phylogeny comprising most freshwater plant lineages; (b) exploited a wide array of functional traits that are important to macrophyte autoecology or that relate to lake ecosystem functioning; (c) assessed if different large-scale beta diversity patterns show a clear latitudinal gradient from the equator to the poles using null models; and (d) employed evolutionary and regression models to first identify the degree to which the studied functional traits show a phylogenetic signal, and then to estimate community- environment relationships at multiple spatial scales. Our results supported the notion that ecological niches evolved independently of phylogeny in macrophyte lineages worldwide. We also showed that taxonomic and phylogenetic beta diversity followed the typical global trend with higher diversity in the tropics. In addition, we were able to confirm that species, multi-trait and lineage compositions were first and foremost structured by climatic conditions at relatively broad spatial scales. Perhaps more importantly, we showed that large-scale processes along latitudinal and elevational gradients have left a strong footprint in the current diversity patterns and community-environment relationships in lake macrophytes. Overall, our results stress the need for an inte- grative approach to macroecology, biogeography and conservation biology, combining multiple diversity facets at different spatial scales. 


\section{Highlight}

-We are beginning to estimate global de-terminants of macrophyte diversity patterns.

- We examine variation of taxonomic, functional and phylogenetic beta diversity.

- Taxonomic and phylogenetic beta diversity is higher in the tropics.

- Functional traits evolve independently of phylogeny in macrophytes worldwide.

- Latitude and elevational gradients left a strong footprit in beta diversity.

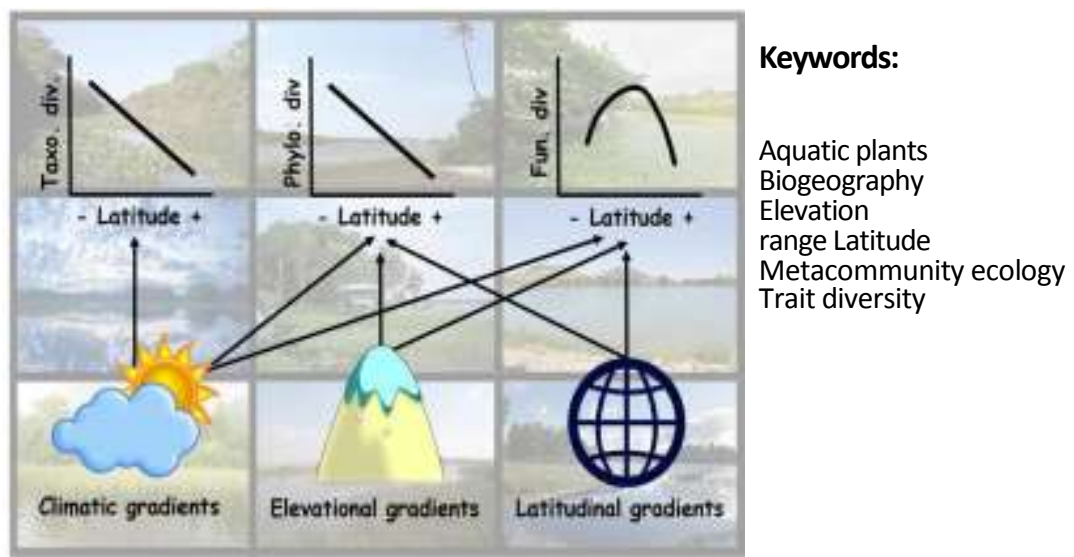

Graphical abstract 


\section{Introduction}

Unravelling the mechanistic basis of large-scale biodiversity patterns has been a major research objective for ecologists and biogeographers for decades. Explanations for these patterns stem from the combined effect of evolutionary, historical and climatic factors such as the last glacial maximum, past dispersal barriers and the latitudinal variation of energy availability (Willig et al., 2003). Although the need to understand large-scale patterns is widely recognised, for example, in the context of niche conservatism (Alahuhta et al., 2017a) and ecosystem functioning and resilience (Pessarrodona et al., 2019), most studies in this research field focus on well-known, and often charismatic, terres-trial vertebrates and woody plants (Moles et al., 2008; Swenson et al., 2012). Instead, other organisms and ecosystems have thus far received considerably less attention (see Heino, 2011 for a comprehensive review). When it comes to the diverse group of freshwater plants, such research program is still in its infancy. More specifically, despite Darwin's (1872) early intuition that the geographical distribution of freshwater plants cannot be accounted by the physical harshness of the environ-ment, it was not until the 21st century that we began generating quantitative estimates of how macroecological gradients affect macrophyte diversity patterns (e.g. Les et al., 2003; Chambers et al., 2008; Alahuhta et al., 2017b; Alahuhta et al., 2018; Murphy et al., 2019). However, obtaining such estimates has been hampered by the availability and low quality of functional and phylogenetic data.

Much of our current understanding of global biodiversity patterns comes from analyses of species diversity data. However, biodiversity is a complex multi-faceted concept that embraces many aspects of biological variation, ranging from phenetic and taxonomic differences to functional complementarity among species (Meynard et al., 2011). Most commonly, species-based beta diversity (SBD), i.e. compositional differences between communities (Whittaker,1960), has been used as a surrogate for all these different facets. An important source of criticism to this traditional approach is that it is silent on functional and phylogenetic differences among species (Devictor et al., 2010), thereby leading to potentially spurious conclusions about large-scale biodiversity patterns. A persistent focus on taxonomic diversity may thus compromise our ability to untangle the mechanistic basis linked to the spatial and temporal dynamics of biodiversity (McGill et al., 2006).

To address these limitations, Graham and Fine (2008) first proposed phylogenetic beta diversity $(P \beta D)$ as a measure of the evolutionary dissimilarity between communities, providing a more comprehensive framework to explain the role of historical imprints on community membership and how evolutionary legacy underlies patterns of niche divergence (Mouquet et al., 2012). Meanwhile, functional beta diversity (FBD), reflecting the morphological, ecological and physiological similarities between communities (Loiseau et al., 2017), was shown to better explain ecosystem functioning and species niche relationships than other classical measures of species diversity (GarcíaGirón et al., 2019a; Jones et al., 2019). Recently, there have been several calls (e.g. Cadotte et al., 2019) to better understand whether ecologically relevant functional traits are conserved along the phylogeny or not (i.e. whether or not phylogenetic signals exist in the functional traits important for coexistence). To do this, phylogenetic signal is typically addressed by testing if closely related species share more similar traits than expected by chance (Blomberg et al., 2003). Although this original framework has been criticised by some authors (e.g. Mouquet et al., 2012), phylogenetic signal tests still provide comprehensive insights into community assembly by, for example, helping to evaluate hypotheses about niche similarities and evolution (Cadotte et al., 2019).

It is now becoming clear that a simultaneous analysis of several diversity facets should provide the most basic building blocks to embrace the multifaceted nature of biodiversity and, at the same time, reveal aspects of community assembly beyond inferences that can be drawn from more traditional species-centred approaches (Jones et al., 2019). This reasoning is especially true from a conservation perspective. Indeed, mapping the imprint of functional and evolutionary processes on beta diversity has been recently proposed as an additional and promising tool to improve large-scale conservation planning in the long run (Devictor et al., 2010), allowing environmental managers and conservation practitioners to focus not only on individual species rarity and distinctness, but also on ecosystem functioning and evolutionary legacy (Owen et al., 2019).

Here, for the first time, we examine the spatial variation of $S \beta D, F B D$ and $\mathrm{P} \beta \mathrm{D}$ using lake macrophyte data sets from 16 regions worldwide (Fig. 1a). To do this, we built the very first phylogeny comprising most freshwater plant lineages and examined functional traits that are important to macrophyte autoecology or that relate to lake ecosystem functioning. More specifically, we studied (i) if a phylogenetic signal is found in the functional traits of macrophytes on a global scale; (ii) if different large-scale beta diversity patterns show a clear latitudinal gradient from the equator to the poles; (iii) if $S \beta D, F \beta D$ and $P \beta D$ respond to similar ecological drivers (i.e. local environment, geography and climate) involved in community assembly (i.e. within-region approach); 


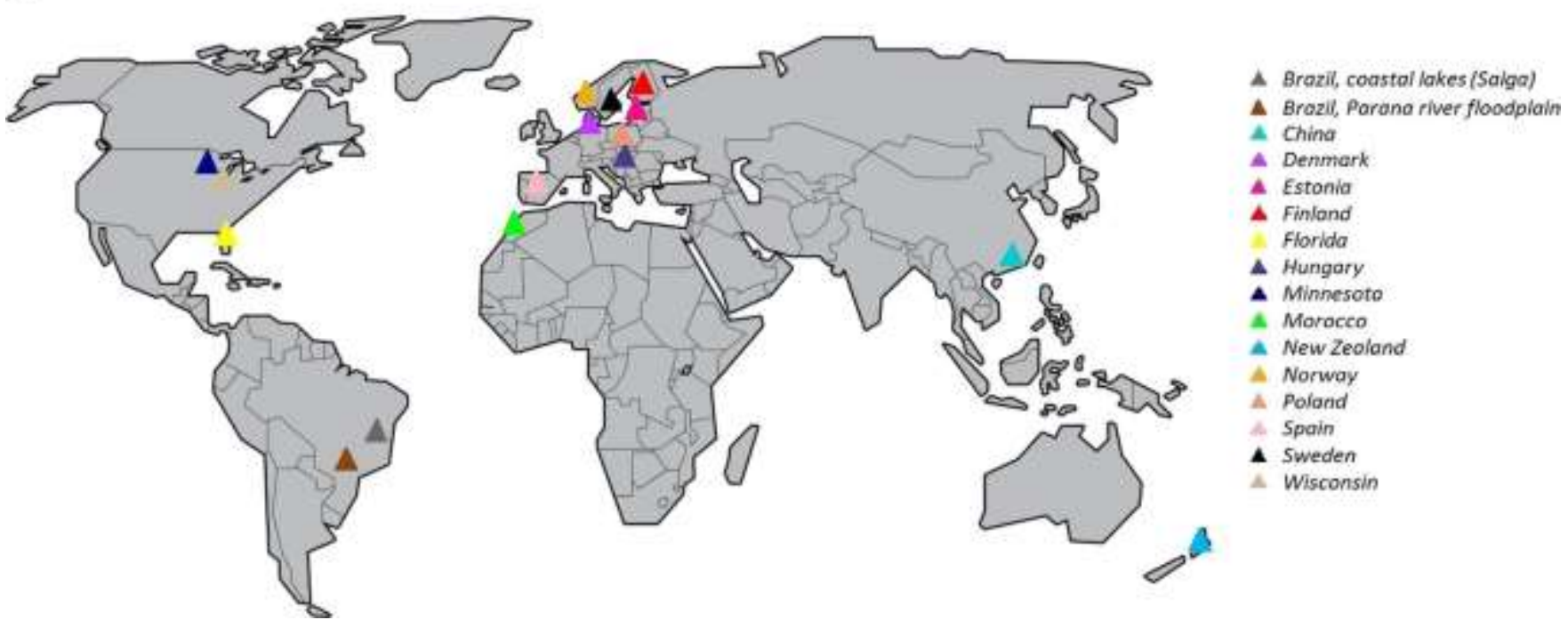

b
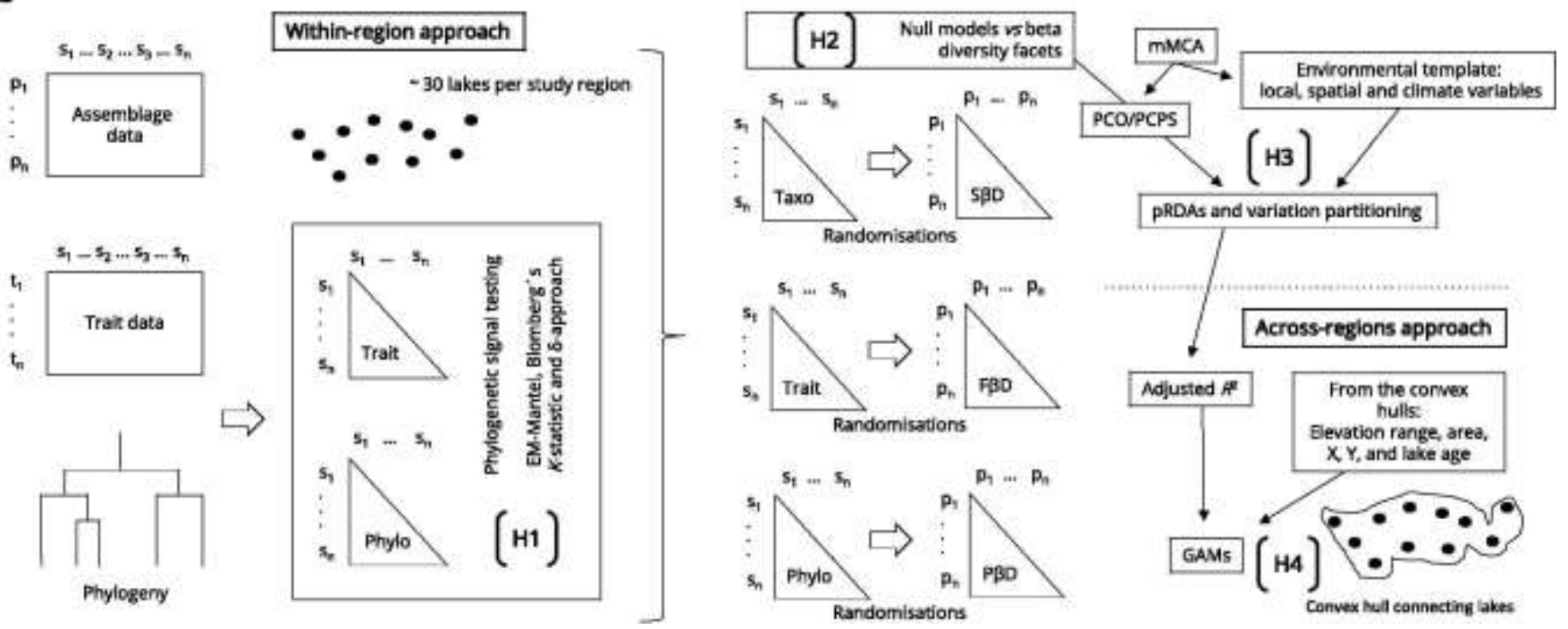

Fig. 1. (a) Our study system comprised ca. 30lakes surveyed in 16 regions (coloured triangles) across the world. (b) A flow chart of the statistical analyses used. We identified (i.e. withinregion approach) the degree to which the studied functional traits showed a phylogenetic signal. For each beta diversity dimension (i.e. taxonomic, functional and phylogenetic), we calculated the mean overall value and tested whether the mean value was different from that expected by chance using null models. We also investigated macrophyte beta diversity patterns in relation to local variables, geographical coordinates and climate variables for each metacommunity using a combination of partial redundancy analysis (pRDA), variation partitioning and multivariate multi-scale codependence analysis (mMCA). Adjusted $\mathrm{R}^{2}$ values from the pure fractions of variation partitioning (i.e. pure local, spatial and climate variables, and the full model including all variables) were regressed in the across-regions approach against a set of environmental variables (i.e. elevation range, area, geographical coordinates, and estimated maximum lake age) obtained from a convex hull that connected all 30 lakes in a region. See the main text for details. $\mathrm{s}$, species; $\mathrm{p}$, sites; $\mathrm{t}$,

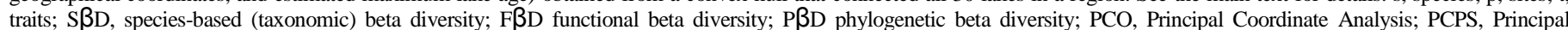
Coordinates of Phylogenetic Structure; X, mean longitude of convex hull; Y, mean latitude of convex hull; and GAMs, generalised additive models; H, hypotheses (see the main text). (For interpretation of the references to colour in this figure legend, the reader is referred to the web version of this article.)

and (iv) how well community-environment relationships across multiple metacommunities (i.e. across-regions approach) can be accounted for by classical ecogeographical gradients (i.e. elevation range, spatial extent, latitude, longitude, and age of the oldest lake within each region; Fig. 1b). Based on a previous study on species niche conservatism for local macrophyte communities (Alahuhta et al., 2017a), we expected to find some level of phylogenetic signal across the study regions $(\mathrm{H} 1)$. However, as rates of trait evolution and speciation may differ among regions (Mouquet et al., 2012), biogeographical comparisons would reveal different degrees of phylogenetic signals. Following Rapoport's rule (Stevens, 1989) that species geographical ranges and niche width should increase at higher latitudes, giving rise to a decrease in beta diversity (Soininen et al., 2007), we expected that SßD, FßD and PßD would show a clear decreasing latitudinal trend ( $\mathrm{H} 2)$. In addition, we assumed following Poff (1997) that large-scale climatic variables (e.g. mean temperature and precipitation) would first select macrophytes that have traits matching the environment, and then these traits would subsequently be sorted by smaller-scale environmental drivers (e.g. water transparency and water total phosphorus concentration; $\mathrm{H3}$ ). Because latitude and elevation range were found to structure macrophyte diversity patterns in previous studies (e.g. Jones et al., 2003; Alahuhta et al., 2017b; Alahuhta et al., 2018), we expected that the two predictors would significantly account for variation in SBD, FBD and $\mathrm{PBD}$ across regions (H4). 


\section{Materials and methods}

\subsection{Test data set}

The macrophyte data set and field methods have been described previously (Alahuhta et al., 2017b, 2018), and we thus only mention the specific details here. We compiled lake macrophyte data for 16 different regions (ca. 30 study lakes within each region) with variable sizes around the world (Fig. 1a and Supporting Information Appendix S1, Fig. S1). The selected lakes ranged from glacial-origin relatively stable lakes situated in temperate and boreal zones (e.g. Sweden and US states of Minnesota and Wisconsin) to semi-arid shallow Mediterranean lakes (e.g. Morocco and Spain), but all were influenced by anthropogenic pressures to varying degrees (e.g. water-level fluctuations, alien invasive species, nutrient enrichment, and decreased connectivity). The species presence-absence data were obtained during field surveys using broadly the same procedures within each study region (Supporting Information Appendix S1, Table S2).

\subsubsection{Explanatory data: within-region approach}

Local variables comprised lake area $\left(\mathrm{km}^{2}\right)$, water transparency measured by Secchi depth $(\mathrm{m})$ and water total phosphorus concentration ( $\mathrm{mg} \mathrm{l}^{-1}$ ), and were surveyed and determined following similar protocols across the regions (Supporting Information Appendix S1). Climate variables included atmospheric annual mean temperature $\left({ }^{\circ} \mathrm{C}\right)$, annual temperature range $\left({ }^{\circ} \mathrm{C}\right)$, and annual precipitation $(\mathrm{mm})$ defined for each study lake based on 30 years average values ( $1 \mathrm{~km}$ resolution data) obtained from the WorldClim (Hijmans et al., 2005). These variables were chosen because they often account for significant variation in community structure of freshwater plants and correlate with other hydromorphological and water chemistry variables that were not consistently available for all study regions (see Alahuhta et al., 2018). Geographical coordinates of lake centres were used to represent spatial locations within each region. We utilised geographical coordinates alone because we wanted to balance the study design by including the same number of predictors in each of the three lake-level explanatory variable groups.

\subsubsection{Explanatory data: across-regions approach}

We used convex hulls to delineate the minimal area containing all surveyed lakes within a region (Alahuhta et al., 2017b). We then used the convex hulls to extract environmental information for each region: (i) elevation range within the convex hull (m); (ii) area of the convex hull $\left(\mathrm{km}^{2}\right)$; (iii) latitude of the convex hull (from centroid); (iv) longitude of the convex hull (from centroid); and (v) estimated maximum lake age within each region (i.e. a ranked variable ranging from the youngest to the oldest lake, see Supporting Information Table S1). These variables can indirectly affect freshwater plants by indicating variation in habitat suitability, dispersal limitation (García-Girón et al., 2019b), large-scale climate and natural geological properties, and temporal availability for colonisation sources (Alahuhta et al., 2017b).

Further details on the test data set can be found in Supporting Information Appendix S1, Tables S1 and S2.

\subsection{Trait and phylogenetic information}

We selected a total of 10 key functional (including morphological and life history) traits to provide information on the features that could potentially be selected by environmental filters (see Supporting Information Appendix S2 for details). To clarify the covariance structure and relative adaptive significance of each functional trait, we applied the orthogonal rotation approach (PCAmix) implemented in the R package PCAmixdata (Chavent et al., 2017). For the following analyses, we used the mixed-variables coefficient of distance (i.e. a generalization of Gower's distance) to extract a trait distance matrix, which described the functional dissimilarity between all species pairs based on a suite of traits with the largest squared loadings on the first two PCAmix axes. The functional dissimilarity matrix was obtained with the 'vegdist' function of the vegan package (Oksanen et al., 2016) in $R$ 3.4.4 (R Development Core Team, 2018).

We analysed 310 nucleotide sequences in two regions from the chloroplast DNA - cpDNA (rbcL - 542 bp and matK - 749 bp) to build the very first phylogeny comprising 71 freshwater plant genera. These sequences were retrieved from GenBank and aligned by Clustal-W using multiple sequence alignment (MSA). Since cpDNA has a relatively slow rate of nucleotide substitutions, we had no problem to obtain an unambiguous alignment. The maximum likelihood (ML) method from the MEGA 5 software (Tamura et al., 2011) was used to create the phylogenetic trees. The optimal substitution model was determined for each locus based on the Akaike Information Criterion - AICc (rbcL, Tamura 3-parameter + Gamma distribution with Invariant sites; matK, General Time Reversible + Gamma distribution with Invariant sites) and the reliability of each branch was tested using the bootstrap method with 1000 replications. The molecular data provided a fully resolved genus-level phylogeny for lake macrophytes but failed to resolve evolutionary relationships within species groups (Fig. 2). We transformed the phylogenetic tree to ultrametric and subsequently estimated the phylogenetic dissimilarity matrix using the 'force. ultrametric' and 'cophenetic' functions from the packages phytools (Revell, 2019) and stats (R Development Core Team, 2018), respectively.

\subsection{Testing for a phylogenetic signal in functional traits}

The degree to which the studied functional traits showed a phylogenetic signal was assessed by means of a two-step approach consisting of a standard Mantel test followed by a test based on the Brownian motion model (i.e. EM-Mantel, Debastini and Duarte, 2017). One of the main advantages of EM-Mantel for estimating phylogenetic signal in a wide array of functional traits is that it allows the use of multiple types of variables (here, continuous and categorical, Supporting Information Appendix S2) simultaneously. The null hypothesis of no phylogenetic conservatism was tested for significance using 999 random permutations with the original $R$ code provided by Debastini and Duarte (2017). Likewise, the strength of phylogenetic signal for individual continuous traits (Supporting Information Appendix S2) was estimated with Blomberg's K-statistic (Blomberg et al., 2003) using the 'multiPhylosignal' function in the picante package (Kembel et al., 2010). This test compares the variance of the phylogenetically independent contrast of the trait studied against those obtained with data randomly reshuffled across the tips of the phylogeny (Blomberg et al., 2003). We also implemented the recently-developed $\delta$-approach of Borges et al. (2019) to unravel the degree of phylogenetic signal of the individual categorical traits (Supporting Information Appendix S2). This approach first calculates the node entropies using a linear version of the Shannon entropy and then implements a Bayesian inferential scheme including two Metropolis-Hastings steps in a Gibbs sampler algorithm (Borges et al., 2019). Under this framework, the higher the $\delta$ value, the greater the quantity of information by the ancestral inferences (i.e. the higher the degree of phylogenetic signal of the categorical trait).

\subsection{Measuring SßD, FßD and PßS}

We first assessed $S \beta D, F \beta D$ and $P \beta D$ by comparing estimates of dissimilarity between all pairs of sampled lakes in each study region to expected levels of dissimilarity under null models. Specifically, we estimated S $\beta D$ using a modified Raup-Crick dissimilarity metric (Raup and Crick, 1979). This metric tests the probability of two communities of a given species richness being more or less dissimilar from the null expectation. The null expectation was generated using 10,000 randomizations with the 'raupcrick' function in the vegan package. 
a

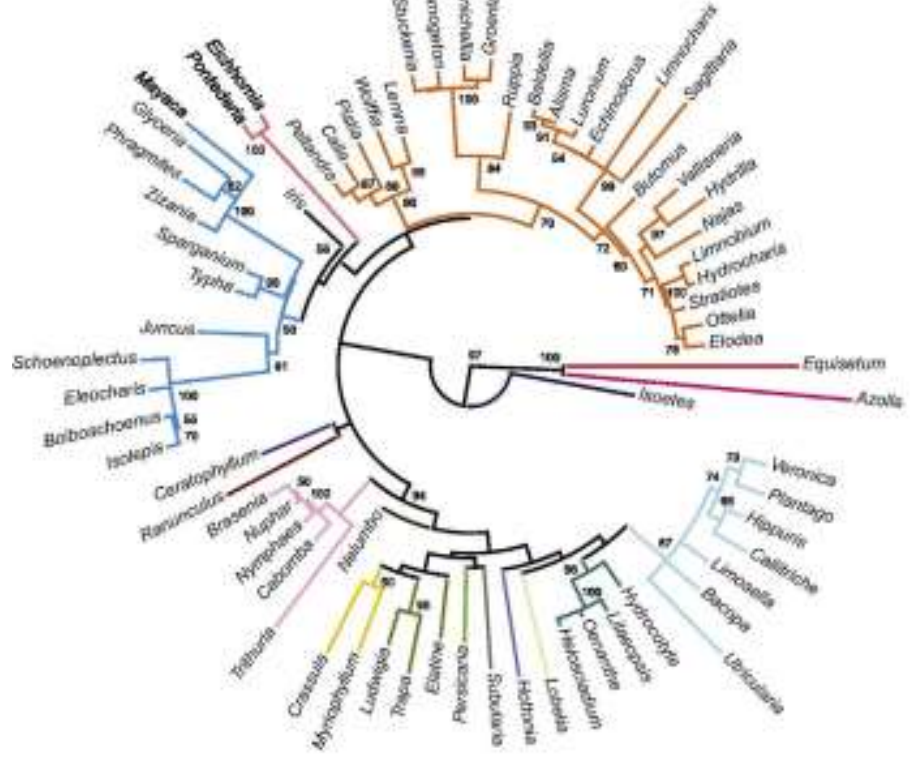

b

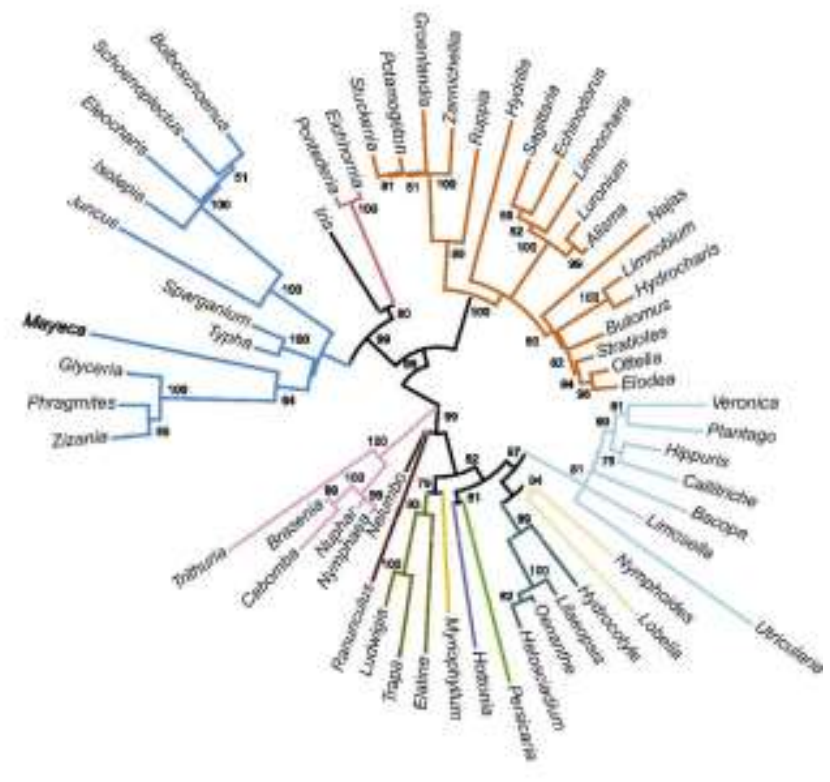

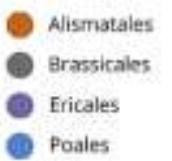
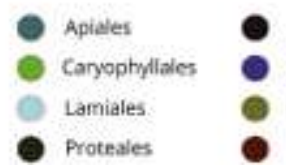

Asparagales

Ceratophyllaies

Malpighiales

Hanunculaies

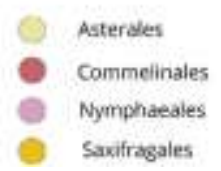

Fig. 2. Maximum likelihood phylogenetictree based on (a) rbcL and (b) matKsequences. Numbers at nodes representthe proportion (\%) of 1000 bootstrap replicates supporting each node. Bootstrap values of $<50 \%$ are not included. Note that the rbcL tree provides an overall topology similar to that seen in the matKtree. However, since the rbcLsequence data cover a total of 71 different plant genera (and the matK sequence data cover 62 plant genera), we only retained the former phylogenetic tree for comparative analyses.

For trait and phylogenetic measures, we estimated the mean overall value using mean pairwise distance (MPD) indices (i.e. Swenson, 2014). To do this, we randomised the identities of species in the functional distance matrix and phylogenetic tree, respectively, and then maintained the species richness of each lake to generate 10,000 random communities. After each randomisation, FßD and PßD were calculated for each study region and these values were used to compute the null distribution. Using these metrics, we obtained the standardised effect size (SES) as:

$\mathrm{SES} 1 / 4$

mpd.obs-mpd.rnd.mean

$$
\text { mpd.rnd.sd }
$$

where mpd.obs is the observed ß-diversity, mpd.rnd.mean the mean of the null distribution and mpd.rnd.sd the standard deviation of the null model.

The mean pairwise distances are scaled between -1 and 1 , with 0 being no different than the random expectation; 1 indicating that deterministic environmental filters favour dissimilar species compositions among sites; and -1 meaning that assemblages comprise functionally similar or closely related species (Swenson, 2014). To test whether the mean overall values of SßD, FßD and PßD were different from the expected value of zero for random data, we performed a series of twotailed Wilcoxon signed-rank tests using $R$ statistical software.

Prior to direct gradient analyses and regression-based modelling, we created a matrix of orthogonal eigenvectors, each of them describing taxonomic, trait and phylogenetic dissimilarities. To do this, FßD was recalculated with the 'COMDIST' function of the picante $R$ package and PßD was addressed using the phylogenetic fuzzy-weighting method with the Bray-Curtis dissimilarity family as implemented in the PCPS package (Debastiani, 2018). The pairwise output values for SßD and FßD were synthesised into principal coordinate analysis (PCO) with the 'pcoa' function in the ape R package (Paradis et al., 2019). Following
Duarte et al. (2012), we retained as many PCO/PCPS eigenvectors as required to minimise the residual sum of squares when regressing taxonomic, functional and phylogenetic composition to response matrices.

\subsection{Variation partitioning and regression-type analyses}

Under the within-region approach, we applied partial redundancy analyses ( $p R D A$ ) to identify significant variables structuring variation in community composition, functional traits and freshwater plant lineages. To partition variation this way, separate pRDA analyses were conducted for the 16 study regions using as response matrices: (i) the PCO eigenvectors describing SßD; (ii) the PCO eigenvectors describing FßD; and (iii) the PCO eigenvectors (i.e. principal coordinates of phylogenetic structure, PCPS) describing patterns of PßD. Biotic variation was partitioned into three independent and four shared fractions: pure local variables; pure spatial variables; pure climate variables; their shared fractions; and residual fraction of unexplained variation (Legendre and Legendre, 2012). The proportion of variation explained by each of the three variable groups was evaluated using adjusted R2 values (adjR2; Peres-Neto et al., 2006). All ecological variables were forced in the analyses to maintain comparability among the 16 study regions and gain equal amount of information for the across-regions approach.

pRDAs isolate the respective effects of local conditions, space and climate, but spatial structures can take several shapes and may not be related exclusively to dispersal. Such kind of information would be highly complementary to variation partitioning by providing explanations for any combined effects of space and the environment (sensu Poff, 1997). To examine more specifically the spatial structures generated by the joint variation in the environment and community composition in each study region, we performed multivariate multi-scale codependence analysis (mMCA, Guénard and Legendre, 2018). The significance of the spatial codependence between PCO/PCPS describing different 
beta diversity facets and environmental variables was assessed through permutation tests using the 'permute.cdp' function from the codep package (Guénard et al., 2018). We also performed mMCA on community-weighted means (CWMs) of transformed trait scores and ran single value decomposition (SVD) to map relationships between traits, environmental descriptors and spatial scales in niche space (see Guénard and Legendre, 2018 and vignettes available in Guénard et al., 2018). All environmental variables were centred and standardised to unit variance prior to conducting the analyses.

In the across-region approach, we used generalised additive models (GAM) with a quasipoisson error distribution (logarithmic link function) to analyse responses of adjR2 values obtained from the pure and shared fractions of variation partitioning to individual environmental gradients (i.e. elevation range, area, latitude, longitude, and estimated maximum lake age within convex hulls). Smoothness selection was estimated with generalised cross validation (GCV) and diagnostic information about the fitting procedure and the convergence of the smoothness selection optimisation was calculated using the 'gam. check' function. We focused on the percentage of deviance explained to identify the goodness-of-fit of the full models, while the relative contribution of each predictor to the final model was assessed as the drop of deviance explained by the model when the variable was removed. Model fitting was accomplished using the mgcv R package (Wood, 2018). See Fig. 1b for the main steps of the statistical approach.

\section{Results}

Factorial analysis of mixed data (PCAmix) clarified the covariance structure of trait variables, suggesting that all functional traits were likely to influence how freshwater plant species might respond to environmental constraints (Supporting Information Appendix S3). The general Mantel test revealed a strong correlation between trait and phylogenetic distances only in five out of 16 study regions. This likely underlined the low phylogenetic signal of the functional traits when all study regions were combined (Table 1). In addition, phylogenetic conservatism differed when functional attributes were considered separately, with some traits (e.g. offspring size) having relatively strong signals across individual regions (Supporting Information Appendix S4). However, the measured functional traits, both considering individual

Table 1

Phylogenetic signal (EM-Mantel; standard Mantel - R, and Brownian motion simulation - Null K) for macrophyte functional traits in each study region. Significant results (*p $0.05 ; \cdots$ p $<0.01$ ) are marked in bold.

\begin{tabular}{cc}
$\begin{array}{l}\text { General Mantel } \\
\text { test }\end{array}$ & $\begin{array}{l}\text { Brownian motion } \\
\text { simulation }\end{array}$ \\
\hline $\mathrm{R}$ & $\mathrm{NK}$
\end{tabular}

\begin{tabular}{lll}
\hline Global data set & 0.12 & \\
Regions & & \\
Brazil, coastal lakes & 0.08 & \\
Brazil, Paraná river floodplain & 0.05 & \\
China & 0.21 & 0.9 \\
Denmark & 0.05 & \\
Estonia & $0.20^{*}$ & \\
Finland & 0.06 & 0.9 \\
Florida & 0.09 & \\
Hungary & $0.22^{*}$ & \\
Minnesota & 0.14 & \\
Morocco & 0.12 & 0.9 \\
New Zealand & 0.07 & 0.9 \\
Norway & 0.06 & \\
Poland & $0.17^{*}$ & 0.9 \\
Spain & $0.17^{*}$ & \\
Sweden & 0.11 & \\
Wisconsin & $0.27^{* *}$ & \\
\hline
\end{tabular}

Note that if, and only if, the standard Mantel test is significant ( $<<0.05)$, the second step assesses whether such correlation between phylogeny and functional attributes is higher than what would be expected by chance given a Brownian motion evolutionary model. regions and together combining all regions, were not likely to be more conserved along the phylogeny than what would be expected by a Brownian motion evolutionary model (Table 1).

Functional beta diversity varied from 0.11 in New Zealand to -0.52 in Norway, whereas the highest taxonomic and phylogenetic dissimilarities were both found in Brazil's coastal lakes (0.93 and 0.08 , respectively). By contrast, the lowest taxonomic and phylogenetic mean values occurred in Estonia (0.40) and Denmark $(-0.31)$, respectively. Based on null models, SBD was different from that expected by chance in all study regions, whereas the mean values for $F \beta D$ and $P \beta D$ were not different from the expected value of zero (Fig. $3)$, which means that only $S \beta D$ refers to any kind of deterministic filtering process across the sites. Importantly, SBD $\left(R^{2}=-0.69 ; p<\right.$ $0.01)$ and $P \beta D\left(R^{2}=-0.51 ; p=0.03\right)$ decreased significantly with increasing latitude, whereas $F \beta D$ showed higher degree of functional redundancy in tropical and high-latitude regions (Fig. 3).

Only the first two PCO/PCPS eigenvectors were selected as response variables for taxonomic, functional and phylogenetic analyses (Supporting Information Appendix S5). Of the pure fractions, local variables were significant in three, five and six regions out of 16 for taxonomic, functional and phylogenetic community structure, respectively (Table 2 ). Pure spatial variables were important in one region out of 16 in taxonomic and functional analyses, respectively, whereas they contributed significantly to phylogenetic community structure in five regions. Pure climate effects were significant in three, two and five regions out of 16 for taxonomic, functional and phylogenetic community structure, respectively. Overall, the joint influence of all the three variable groups on $S \beta D, F B D$ and $P \beta D$ was highly important in Norway, the US state of Wisconsin and Spain, respectively. Other joint effects also showed high-explained variation in Brazil's Paraná river floodplain, Estonia, Florida, Minnesota, Morocco, Norway and Sweden (Supporting Information Appendix S6).

Forward selection identified different significant variables structuring $S \beta D, F B D$ and $P \beta D$ in each study region (Supporting Information Appendix S7). The mMCA application revealed different components of the spatial codependence between macrophyte beta diversity patterns and environmental variables (Supporting Information Appendix S8). Clearly, the strongest component associated climate variables with $S \beta D, F \beta D$ and $P \beta D$ at relatively broad spatial scales. The combination of these spatial codependence effects highlights that a large amount of species, functional and evolutionary variation occurs in some regions along a temperature gradient (e.g. China, Hungary, Minnesota) and along a precipitation gradient (e.g. Morocco, Poland) at relatively broad spatial scales, and along local environment gradients (e.g. Brazil's Paraná river floodplain, New Zealand, Spain) at finer spatial scales. Our results further revealed the association of climatic gradients and leaf dry mass content (LDM) at relatively broad spatial scales in nine out of the 16 regional metacommunities. Similarly, we found some evidence for the relationships between recruitment traits (i.e. offspring size) and specific leaf area (SLA) with climate and local environ-mental gradients at different spatial scales. However, trait-environment relationships remained relatively dissimilar among the study regions (Supporting Information Appendix S8).

The 'best' GAM models modestly explained different beta diversity patterns across metacommunities (Table 3 ), probably due to the number of study regions $(N=16)$. None of the regional explanatory variables accounted for variation in any pure or joint fraction of S $\beta D$. By contrast, regression models selected the following regional descriptors to explain FBD and PBD: latitude, elevation range and longitude. Clearly, the effects of latitude were highly significant in explaining the joint and pure local fractions of $F \beta D$ and $P \beta D$. The association between latitude and the full model explaining $F \beta D$ was positive, whereas the partial response curves indicated a negative relationship of latitude with the joint and pure local fractions explaining lineage composition (Fig. 4). Elevation range was also a highly important predictor for the pure local fraction of $P \beta D$, showing a negative relationship. Similarly, elevation range 

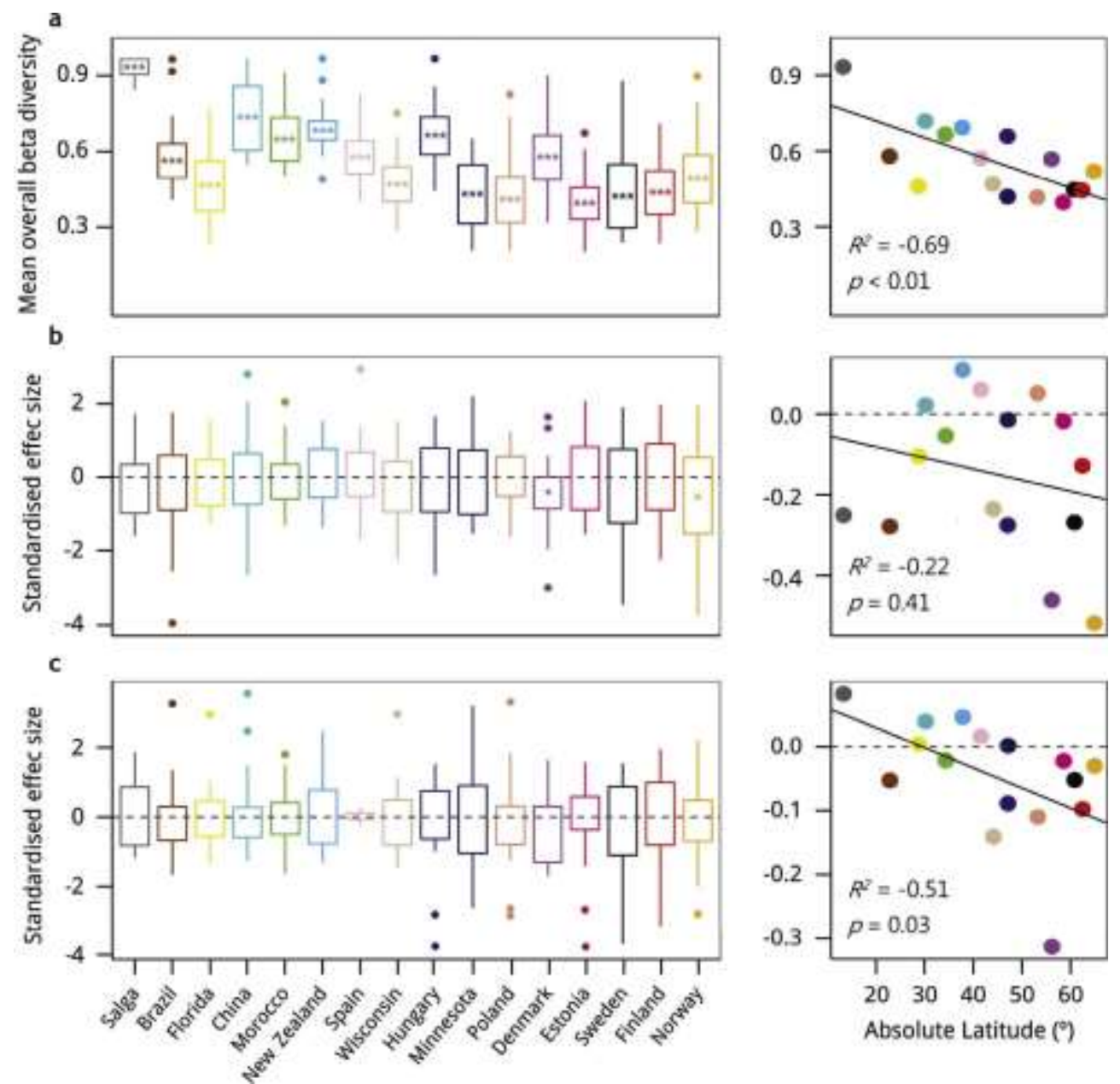

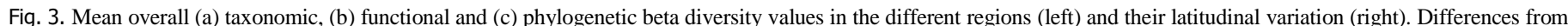

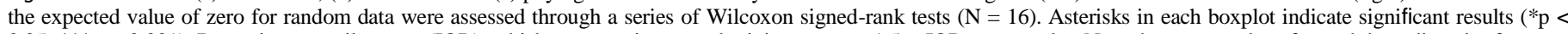

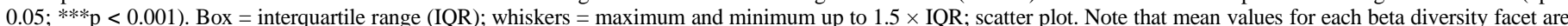

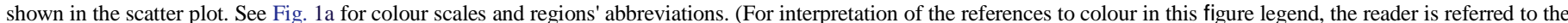
web version of this article.)

Table 2

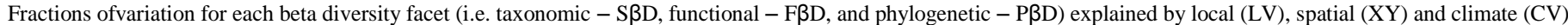
variables in each study region.

Values represent adjusted $\mathrm{R}^{2} \times 100$ based on partial redundancy analysis (pRDA). Significant results (*p $<0.05 ; * * \mathrm{p}<0.01$; ***p $\left.<0.001\right)$ are marked in bold.

\begin{tabular}{|c|c|c|c|c|c|c|c|c|c|c|c|c|}
\hline \multirow[t]{2}{*}{ Regin } & \multicolumn{4}{|l|}{$\mathrm{S} \beta D$} & \multicolumn{4}{|l|}{$\mathrm{F} \beta \mathrm{D}$} & \multicolumn{4}{|l|}{$\mathrm{P} \beta \mathrm{D}$} \\
\hline & LV & $X Y$ & $\mathrm{CV}$ & All & LV & $\mathrm{XY}$ & $\mathrm{CV}$ & All & LV & $\mathrm{XY}$ & $\mathrm{CV}$ & All \\
\hline Salga $^{a}$ & $11.4^{*}$ & 7.5 & $11.4^{*}$ & $29.2 *$ & $17.0 *$ & $<0.1$ & $<0.1$ & $40.1 *$ & $19.7 *$ & $<0.1$ & 3.5 & $60.6^{*}$ \\
\hline Brazil $^{\text {b }}$ & 11.0 & 7.2 & 11.0 & 14.5 & 4.2 & 9.5 & $20.1^{*}$ & 12.4 & $26.1 *$ & 8.1 & $19.2^{*}$ & $55.9 * *$ \\
\hline China & 9.8 & 7.2 & 10.9 & 26.1 & $<0.1$ & $<0.1$ & $<0.1$ & $<0.1$ & $17.1^{*}$ & $16.5^{*}$ & 7.5 & $49.4^{*}$ \\
\hline Denmark & 9.6 & 7.0 & 10.9 & 26.4 & $23.2^{*}$ & 1.3 & $<0.1$ & 27.4 & $28.3^{*}$ & 1.3 & 1.2 & 43.8 \\
\hline Estonia & $11.7^{*}$ & 7.2 & 11.0 & $28.0^{*}$ & $22.9 * *$ & $9.5^{*}$ & 6.9 & $36.0 * *$ & 6.2 & $18.7^{* *}$ & $19.9 *$ & $65.8^{* *}$ \\
\hline Finland & 10.4 & 6.8 & 10.2 & 26.4 & 2.1 & $<0.1$ & 3.3 & $23.7^{*}$ & 9.9 & 1.9 & 4.9 & 39.9 \\
\hline Florida & 11.1 & 7.3 & 10.9 & $29.3 *$ & 5.5 & 4.2 & 1.3 & $15.2 *$ & $31.8^{*}$ & 5.5 & 7.9 & $55.9^{*}$ \\
\hline Hungary & 10.0 & 7.9 & 10.6 & 26.5 & $23.8^{*}$ & 6.0 & 3.8 & $36.1^{*}$ & 22.5 & 2.7 & 4.4 & 39.0 \\
\hline Minnesota & 10.2 & 6.7 & 10.0 & 25.7 & 3.5 & $<0.1$ & 4.2 & $28.1^{*}$ & 15.6 & 7.7 & 13.0 & $49.8^{* *}$ \\
\hline Morocco & 10.6 & 6.3 & 10.1 & 26.2 & $<0.1$ & 2.5 & 5.1 & $33.9 * *$ & 9.5 & $16.6^{* *}$ & $20.5^{*}$ & $56.5^{* * *}$ \\
\hline New Zealand & 10.1 & 6.5 & 10.4 & 25.7 & $<0.1$ & 5.9 & 3.2 & $29.3 * *$ & 4.1 & 6.8 & 1.4 & $52.6^{*}$ \\
\hline Norway & $14.5^{*}$ & $12.5^{*}$ & $14.7^{*}$ & $40.3^{* *}$ & 5.2 & 2.1 & $22.1 *$ & $27.8^{*}$ & 9.9 & 8.9 & $15.1^{*}$ & 38.5 \\
\hline Poland & 11.0 & 7.6 & $11.2^{*}$ & $28.2^{*}$ & $<0.1$ & $<0.1$ & $<0.1$ & $32.0 *$ & 9.0 & 7.0 & 7.6 & 43.4 \\
\hline Spain & 10.3 & 6.6 & 10.1 & 25.0 & 7.2 & 0.7 & 9.7 & 14.5 & 10.3 & $23.7^{* *}$ & $31.1^{* *}$ & $68.7^{* * *}$ \\
\hline Sweden & 10.0 & 7.4 & 11.2 & 26.6 & $30.8^{* *}$ & $<0.1$ & $<0.1$ & $48.6 * *$ & $17.9^{*}$ & $11.8^{*}$ & 7.6 & $63.2^{* * *}$ \\
\hline Wisconsin & 10.6 & 7.1 & 10.5 & 25.8 & $<0.1$ & $<0.1$ & $<0.1$ & $53.7 * *$ & 8.4 & 6.2 & 9.6 & $56.3^{* * *}$ \\
\hline
\end{tabular}

Note that separate pRDA analyses using the same explanatory variables were performed for each study area.

${ }^{\text {a }}$ Salga Brazil, coastal lakes.

${ }^{\mathrm{b}}$ Brazil Brazil, Paraná river floodplain. 
Table 3

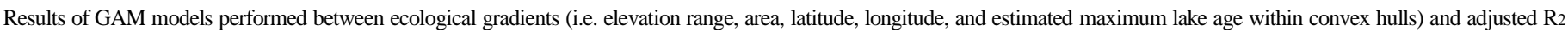

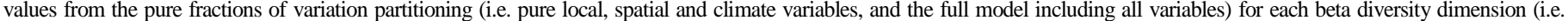
taxonomic - S $\beta D$, functional - F $\beta D$, and phylogenetic $-P \beta D)$. Significant results (*p < 0.05; **p < 0.01) are marked in bold.

\begin{tabular}{|c|c|c|c|}
\hline & \multicolumn{2}{|c|}{ Summary of GAMmodels } & \multirow[t]{2}{*}{ Variables selected (\% drop deviance explained) } \\
\hline & Adj. $R^{2} \times 100$ & Total explained variance $(\%)$ & \\
\hline \multicolumn{4}{|l|}{$\mathrm{S} \beta \mathrm{D}$} \\
\hline Pure local adj. $\mathrm{R}^{2}$ & 2.1 & 24.2 & \\
\hline Pure spatial adj. $\mathrm{R}^{2}$ & 3.6 & 31.0 & \\
\hline Pure climate adj. $\mathrm{R}^{2}$ & 4.7 & 30.2 & \\
\hline Full model adj. $\mathrm{R}^{2}$ & $<0.1$ & 6.2 & \\
\hline \multicolumn{4}{|l|}{$\mathrm{F} \beta \mathrm{D}$} \\
\hline Pure local adj. $\mathrm{R}^{2}$ & 2.1 & 31.9 & \\
\hline Pure spatial adj. $\mathrm{R}^{2}$ & 1.8 & 34.5 & \\
\hline Pure climate adj. $\mathrm{R}^{2}$ & 10.1 & 40.1 & \\
\hline Full model adj. $\mathrm{R}^{2}$ & 21.9 & 48.0 & Latitude $\left(9.2^{* *}\right)$, elevation $\left(6.8^{*}\right)$, longitude $\left(5.1^{* *}\right)$ \\
\hline \multicolumn{4}{|r|}{ 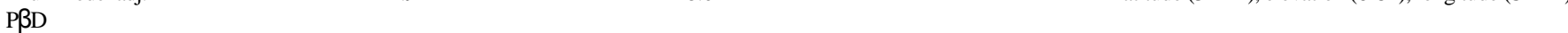 } \\
\hline Pure local adj. $\mathrm{R}^{2}$ & 16.2 & 44.1 & Elevation $\left(13.9^{*}\right)$, latitude $\left(12.7^{*}\right)$ \\
\hline Pure spatial adj. $\mathrm{R}^{2}$ & $<0.1$ & 5.1 & \\
\hline Pure climate adj. $\mathrm{R}^{2}$ & $<0.1$ & 18.7 & \\
\hline Full model adj. $\mathrm{R}^{2}$ & 1.9 & 34.6 & Latitude (13.3*) \\
\hline
\end{tabular}

and longitude were the second and third most important predictors for the full model explaining the functional metric, having a positive and a negative relationship, respectively.

\section{Discussion}

Contrary to our predictions ( $\mathrm{H} 1)$, we found no evidence for phylogenetic niche signals in ecological traits of lake macrophytes across the study regions. Our findings also indicated that species-based beta diversity $(\mathrm{S} \beta \mathrm{D})$ and phylogenetic beta diversity (PBD) exhibited a relatively high degree of spatial congruence on a global scale, especially as compared to functional beta diversity (F $\beta D$ ), decreasing from the equator to the poles. This finding partially supported our second hypothesis $(\mathrm{H} 2)$ that beta diversity follows the typical global trend with higher diversity in the tropics. Our results also revealed that environmental, spatial and climatic variables together determined $S \beta D, F B D$ and $P \beta D$ within each region. More specifically, on the basis of the mMCA results, we were able to confirm our expectations $(\mathrm{H} 3)$ that species, multi-trait and lineage compositions are first and foremost structured by climatic conditions at relatively broad spatial scales. Importantly, our study suggested that latitudinal and elevational gradients together contributed to variation in $F \beta D$ and $P \beta D$ across regions, partially confirming our fourth hypothesis ( $\mathrm{H} 4)$.

Many quantitative studies assessing the phylogenetic niche conservatism hypothesis (e.g. Pavoine et al., 2013; García-Girón et al., 2019a) found little evidence for closely related species to resemble each other's ecological traits more than species randomly drawn from a phylogeny. Blomberg et al. (2003) even suggested that situations where
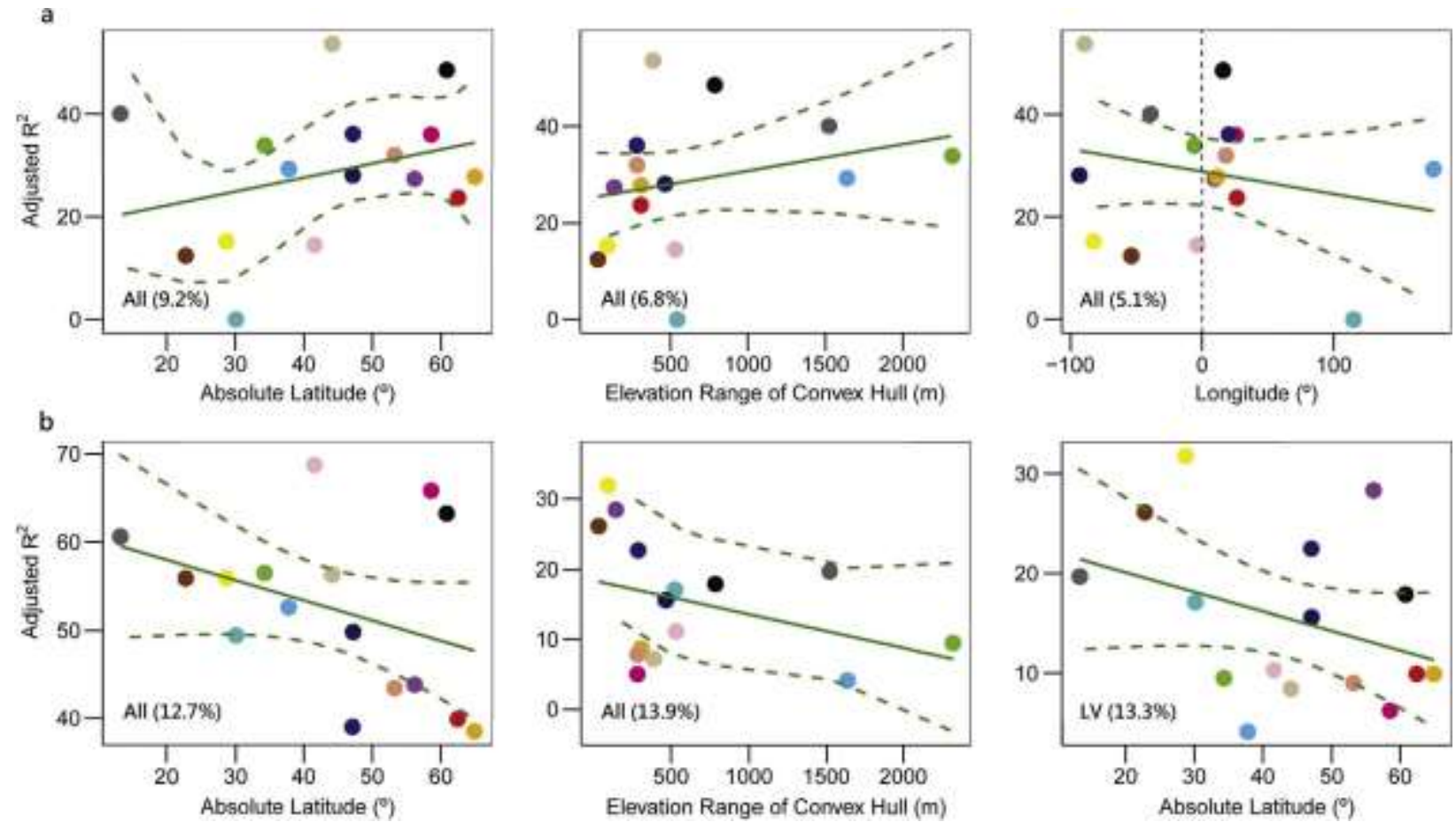

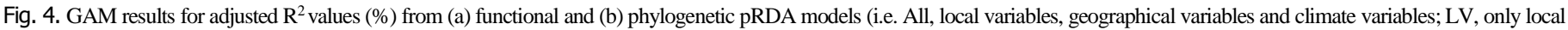

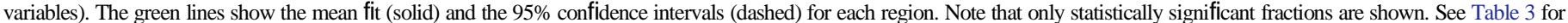
summary statistics $(\mathrm{N}=16)$ and Fig. 1a for colour scales. (For interpretation of the references to colour in this figure legend, the reader is referred to the web version of this article.) 
evolutionary and multi-trait variations are tightly linked may be the exception rather than the rule (but see Cadotte et al., 2019). In our study, these rather weak phylogenetic niche signals were consistent with analyses based on individual traits and together combining all traits, which showed a relatively consistent trend globally (Table 1 ). These findings underscore the idea that ecological niches evolve independently of phylogeny in freshwater plants, which is expectable for functional traits that are convergent in different lineages (e.g. growth form and reproduction mode; Barret et al., 1993) and have high phenotypic plasticity (e.g. specific leaf area and seed length; Zhou et al., 2019). Whatever the case, and despite theoretical criticism of phylogenetic signal analyses (see Mouquet et al., 2012 for a comprehensive review) and the fact that some methodological issues certainly require further attention (e.g. which kind of physiological, morphological and life-history traits are most appropriate for calculating multi-trait distance matrices; see the recent review of Cadotte et al., 2019), our results found no support for the existence of phylogenetic signals in macrophyte lineages worldwide.

Consistent with a growing body of studies (e.g. Gianuca et al., 2018; García-Girón et al., 2019a), we found that local, spatial, and climatic variables together accounted for species, functional and phylogenetic variation within regions. However, we also found some degree of context dependency in the results, which was probably due to differences in environmental gradients among the study regions (Alahuhta et al., 2018). Additional insights into community assembly were provided by the assessment of the effect size of taxonomic, functional and phylogenetic facets. Randomness was by far most commonly detected for FßD and PßD, whereas SßD was sorted primarily by deterministic processes. However, it must be noted that apparent randomness in FßD and PßD may also stem from the combined or antagonistic forces of environmental filtering and biotic interactions (Mayfield and Levine, 2010), and the prevalence of randomness and drift may thus be overestimated in our study. This is likely to be the case because pRDA models and mMCA results (Table 2 and Appendix S7, respectively) found evidence for the role of deterministic environmental filtering on FßD and PßD at different spatial scales. We found, surprisingly, no similar trait-environment relationships across spatial scales in the study metacommunities. Instead, macrophyte trait structure was affected by climatic and environ-mental variation via different processes operating at various spatial scales. However, in more than half of the study regions, we found evidence for the spatial codependence between LDM, a key trait of the leaf economics spectrum representing the average density of leaf tissues (Cornelissen et al., 2003), and climatic gradients at relatively broad spatial scales. This finding may be interpreted as an adaptive strategy reflecting some of the fundamental trade-offs in economics that govern terrestrial and aquatic plants in different biomes (Pierce et al., 2012; Lukács et al., 2017). Perhaps more importantly, our findings suggested that climatic gradients overrode the effects of the local environment on community-weighted means at the largest spatial scales. This partially follows Poff's (1997) idea that large-scale climatic variables (i.e. atmospheric temperature and annual precipitation) first select organisms that have matching speciesspecific traits, and then these sets of species are subsequently sorted by smaller-scale environmental features (i.e. hydromorphological and water quality variables).

The latitudinal diversity gradient - decreasing species richness from the equator to the poles - is one of the best-known macroecological patterns in the natural world (see Kinlock et al., 2018). Strikingly, the spatial patterns observed here for SßD and PßD differed markedly from those that have been observed previously for freshwater plants (Crow, 1993; Alahuhta et al., 2017b; but see a coarse resolution study by Murphy et al., 2019), indicating that these processes may operate differently across spatial scales. We observed that SßD and PßD showed a strong latitudinal gradient similar to those found for a wide variety of organisms, both terrestrial and aquatic (see Hillebrand, 2004; Heino, 2011; and Kinlock et al., 2018 for reviews), thereby supporting modern ideas based on Rapoport's rule (see Soininen et al., 2007). By contrast, FßD patterns were less straightforward than those for SßD and PßD, suggesting a higher degree of functional redundancy in tropical and high-latitude metacommunities, probably as a result of the 'ecological packing' in tropical landscapes and the greater resource limitation near the poles (Willig et al., 2003). Although this point certainly requires further research and attention, it seems to confirm Hutchinson's (1975) intuition that freshwater plant communities are more functionally diverse at temperate latitudes.

The combination of climate and latitude has been found to be a dominant predictor of global patterns of angiosperm plant diversity (Kreft and Jetz, 2007). Meanwhile, Alahuhta et al. (2017b) showed that wider elevation ranges may lead to increasing climate harness that, in turn, affect various aspects of macrophyte morphological and life history traits that are known to be temperature sensitive (e.g. specific leaf area and phenology). However, the association between elevation range and beta diversity may also reflect the greater variability of habitats or resources available with greater variation in elevation, further increasing environmental heterogeneity and thus enabling the colonisation of a greater variety of functionally dissimilar and distantly related species (Alahuhta et al., 2017b). Our GAM results are consistent with these and other studies conducted in the freshwater realm (e.g. Heino, 2011; Alahuhta et al., 2018), supporting our hypothesis that elevation range together with latitude strongly affect the strength of community-environment relationships in the across-regions approach. We thus suggest that a trade-off between climate and habitat diversity may contribute to a robust framework for explaining large-scale diversity patterns and communityenvironment relationships in freshwater plants. The rationale behind this framework is that both factors (i.e. climate and habitat diversity) are strongly linked through latitudinal and elevational variations (Alahuhta et al., 2018). Together, these key driving mechanisms integrate the essential ideas about gradient lengths, macroscale correlations and climate continuity in a single framework that bridges different leading ecological theories (e.g. Brown et al., 2004; Jocque et al., 2010).

\section{Conclusions}

Despite the fact that many hypotheses have been proposed to explain the global patterns of biodiversity, unravelling the mechanisms behind these patterns is one of the major challenges for modern ecology and biogeography. In our study using data on lake macrophyte metacommunities from six continents, we showed how combining different diversity dimensions and spatial scales can provide interesting and complementary results. Despite that we recognised certain deficiencies related to our data sets (e.g. limited numbers of lakes, more regions in Europe than other continents and lack of species-level phylogeny), our findings revealed, for example, that SßD and $\mathrm{P} B D$ exhibited a relatively high degree of spatial congruence on a global scale, especially as compared to FßD, both decreasing from the equator to the poles. Likewise, we found little evidence of phylogenetic niche signals in freshwater plant lineages worldwide. Perhaps more importantly, we showed that large-scale processes along latitudinal and elevational gradients have left a strong footprint in the current diversity patterns and community-environment relationships in lake macrophytes. Overall, our results raised the dilemma of which and how different diversity components should be favoured in large-scale conservation programs. We suggest that implementing and assessing conservation strategies using a given diversity dimension as a cure-for-all should be avoided. Instead, environmental management and conservation biology should benefit from pluralistic approaches combining taxonomic, functional and phylogenetic data. We thus believe that global biodiversity assessments will benefit from integrative approaches connecting biogeography, evolutionary biology and functional ecology at multiple spatial scales 
Jorge García-Girón: Conceptualization, Data curation, Formal analysis, Methodology, Investigation, Writing - original draft, Writing - review \& editing, Funding acquisition, Resources, Validation. Jani Heino: Writing - original draft, Writing - review \& editing, Supervision, Validation, Visualization, Funding acquisition, Resources, Validation. Lars Baastrup-Spohr: Funding acquisition, Resources, Validation. Claudia P. Bove: Funding acquisition, Resources, Validation. John Clayton: Funding acquisition, Resources, Validation. Mary de Winton: Funding acquisition, Resources, Validation. Tônu Feldmann: Funding acquisition, Resources, Validation. Margarita Fernândez-Alâez: Funding acquisition, Resources, Validation. Frauke Ecke: Funding acquisition, Resources, Validation. Patrick Grillas: Funding acquisition, Resources, Validation. Mark V. Hoyer: Funding acquisition, Resources, Validation. Agnieszka Kolada: Funding acquisition, Resources, Validation. Sarian Kosten: Funding acquisition, Resources, Validation. Balâzs A. Lukâcs: Funding acquisition, Resources, Validation. Marit Mjelde: Funding acquisition, Resources, Validation. Roger P. Mormul: Funding acquisition, Resources, Validation. Laila Rhazi: Funding acquisition, Resources, Validation. Mouhssine Rhazi: Funding acquisition, Resources, Validation. Laura Sass: Funding acquisition, Resources, Validation. Jun Xu: Funding acquisition, Resources, Validation. Janne Alahuhta: Conceptualization, Writing - original draft, Writing - review \& editing, Supervision, Validation, Visualization, Funding acquisition, Resources.

Declaration of competing interest

The authors declare no competing financial interests.

\section{Acknowledgments}

JGG appreciates financial support from the Spanish Ministry of Economy and Industry [project METAPONDS, grant CGL2017 84176R], the Junta de Castilla y León [grant LE004G18] and from the Fundación Biodiversidad (Spanish Ministry for Ecological Transition and Demographic Challenge). BAL was supported by National Research, Development and Innovation Fund [grant NKFIH, OTKA PD120775] and by the Bolyai Jânos Research Scholarship of the Hungarian Academy of Sciences. S.K. was supported by NWO Veni [grant 86312012]. Sampling of the coastal Brazilian lakes was financed by NWO [grant W84-549]; The National Geographic Society [grant 7864-5]; and CNPq [grants 480122, 490409, 311427]. We thank the SALGA team, especially Gissell Lacerot, Nestor Mazzeo, Vera Huszar, David da Motta Marques, and Erik Jeppesen for organizing and executing the SALGA field sampling campaign and Bruno Irgang and Eduardo Alonso Paz for helping with identification. We thank Minnesota and Wisconsin Departments of Natural Resources for collecting the macrophyte data. We are grateful to Carol Reschke for her work in combining and performing quality control for the Minnesota macrophyte data used in the analysis. This is contribution no. 607 of the Natural Resources Research Inst. of the Univ. of Minnesota Duluth. Provision of New Zealand macrophyte data was possible via NIWA SSIF funding.

Data accessibility statement

The data sets are from state or national administration, where they can be obtained by request. A key extract of the data set is available in Supporting Information Appendix S1.

Appendix A. Supplementary data

Supplementary data to this article can be found online at https://doi. org/10.1016/j.scitotenv.2020.138021. 


\section{References}

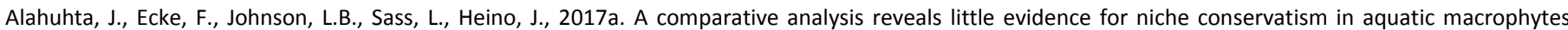
among four areas on two continents. Oikos 126, 136-148.

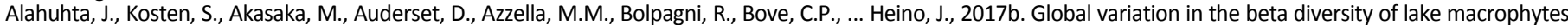
is driven by environmental heterogeneity rather than latitude. J. Biogeogr. 44, 1758-1769.

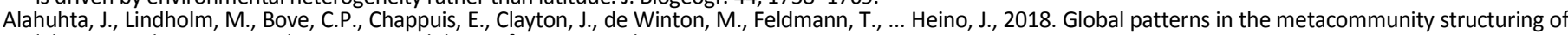
lake macrophytes: regional variations and driving factors. Oecologia 188, 1167-1182.

Barret, S.C.H., Eckert, C.G., Husband, B.C., 1993. Evolutionary processes in aquatic plant populations. Aquat. Bot. 44, 105-145.

Blomberg, S.P., Garland, T., Ives, A.R., 2003. Testing for phylogenetic signal in comparative data: behavioral traits are more labile. Evolution 57, 717-745.

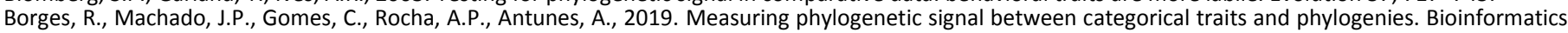
$35,1862-1869$.

Brown, J.H., Gillooly, J.F., Allen, A.P., Savage, V.M., West, G.B., 2004. Toward a metabolic theory of ecology. Ecology 85, 1771-1789.

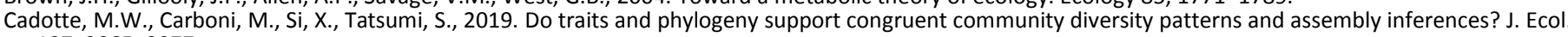
107, 2065-2077.

Chambers, P.A., Lacoul, P., Murphy, K.J., Thomaz, S.M., 2008. Global diversity of aquatic macrophytes in freshwater. Hydrobiologia 595, 9-26.

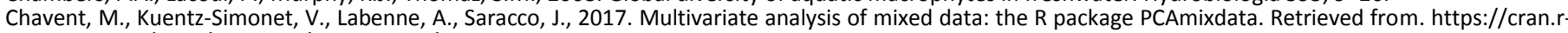
project.org/ web/packages/PCAmixdata/index.html.

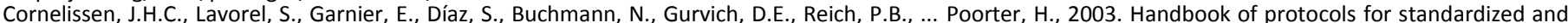
easy measurements of plant functional traits worldwide. Aust. J. Bot. 51, 335-380.

Crow, G.E., 1993. Species diversity in aquatic angiosperms: latitudinal patterns. Aquat. Bot. 44, $229-258$.

Darwin, C.R., 1872. The Origin of Species by Means of Natural Selection. John Murray, London, UK.

Debastiani, V.J., 2018. Package 'PCPS'. Retrieved from. https://cran.r-project.org/web/ packages/PCPS/index.html.

Debastini, V.J., Duarte, L.S., 2017. Evolutionary models and phylogenetic signal assessment via Mantel test. Evol. Biol. 44, $135-143$.

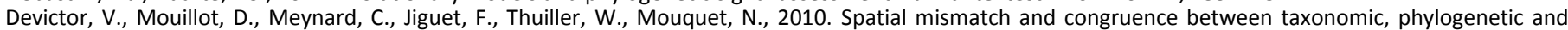
functional diversity: the need for integrative conservation strategies in a changing world. Ecol. Lett. 13, 1030-1040.

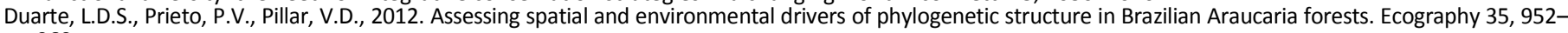
960.

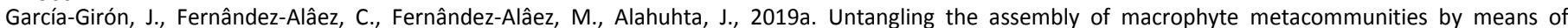
taxonomic, functional and phylogenetic beta diversity patterns. Sci. Total Environ. 693, 133616.

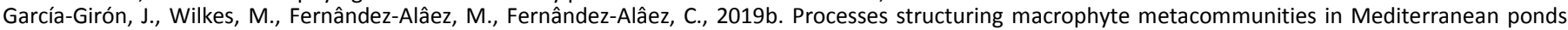
combining novel methods to disentangle the role of dispersal limitation, species sorting and spatial scales. J. Biogeogr. $46,646-656$.

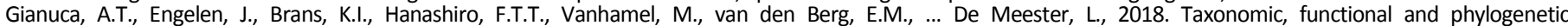
metacommunity ecology of cladoceran zooplankton along urbanization gradients. Ecography 41, $183-194$.

Graham, C.H., Fine, P.V.A., 2008. Phylogenetic beta diversity: linking ecological and evolutionary processes across space in time. Ecol. Lett. 11, $1265-1277$.

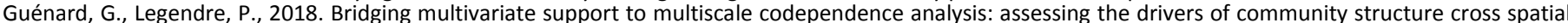
scales. Methods Ecol. Evol. 9, 292-304.

Guénard, G., Legendre, P., Pages, B., 2018. Package 'codep: multiscale codependence anal-

ysis'. Retrieved from. https://cran.r-project.org/web/packages/codep/index.html. Heino, J., 2011. A macroecological perspective of diversity patterns in the

freshwater

realm. Freshw. Biol. 56, 1703-1722.

Hijmans, R.J., Cameron, S.E., Parra, J.L., Jones, P.G., Jarvis, A., 2005. Very high resolution in-

terpolated climate surfaces for global land areas. Int. J. Climatol. 25, 1965-1978. Hillebrand, H., 2004. On the generality of the latitudinal diversity gradient.

Am. Nat. 163,

$192-211$.

Hutchinson, G.E., 1975. A Treatise on Limnology. Limnological Botany vol. 3. John Wiley and Sons, New York, NY, USA

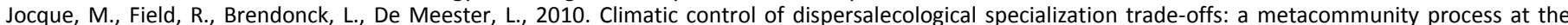
heart of the latitudinal diversity gradient? Glob. Ecol. Biogeogr. 19, 244-252.

Jones, J.I., Li, W., Maberly, S.C., 2003. Area, altitude and aquatic plant diversity. Ecography 26, 411-420.

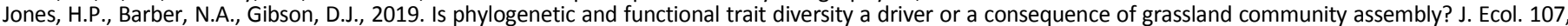
2027-2032.

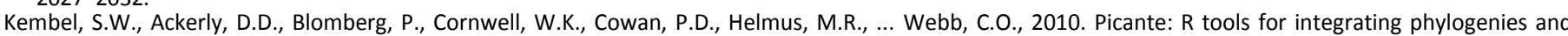
ecology. Bioinformatics 26, 1463-1464.

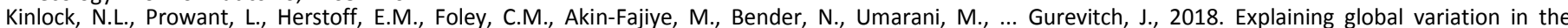
latitudinal diversity gradient: meta-analysis confirms known patterns and uncovers new ones. Glob. Ecol. Biogeogr. 27, 125-141.

Kreft, H., Jetz, W., 2007. Global patterns and determinants of vascular plant diversity. Proc. Natl. Acad. Sci. U. S. A. 104, 5925-5930.

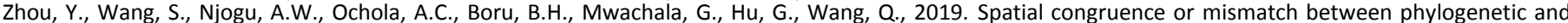
functional structure of seed plants along a tropical elevation gradient: different traits have different patterns. Front. Ecol. Evol. 7, 100.

Legendre, P., Legendre, L., 2012. Numerical Ecology. Elsevier, Amsterdam, The Netherlands.

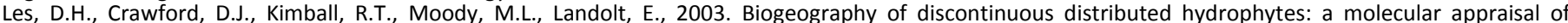
intercontinental disjunctions. Int. J. Plant Sci. 164, 917-932.

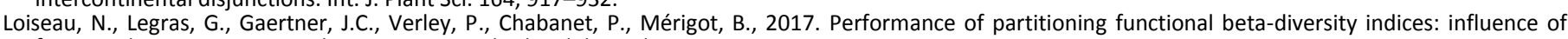
functional representation and partitioning methods. Glob. Ecol. Biogeogr. 26, 753-762.

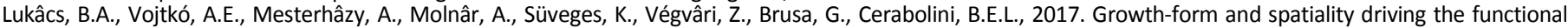
difference of native and alien aquatic plants in Europe. Ecol. Evol. 7, 950-963.

Mayfield, M.M., Levine, J.M., 2010.Opposing effects of competitive exclusion on the phylogenetic structure of communities. Ecol. Lett. 13, 1085-1093.

McGill, B.J., Enquist, B.J., Weiher, E., Westoby, M. 2006. Rebuilding community ecology from functional traits. Trends Ecol. Evol. 21, $178-185$.

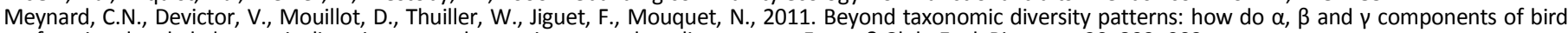
functional and phylogenetic diversity respond to environmental gradients across France? Glob. Ecol. Biogeogr. 20, 893-903.

Moles, A.T., Warton, D.I., Warman, L., Swenson, N.G., Laffan, S.W., Zanne, A.E., Leishman, M.R., 2008. Global patterns in plant height. J. Ecol. 97, 923-932.

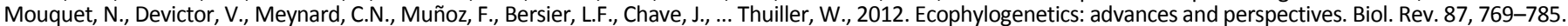

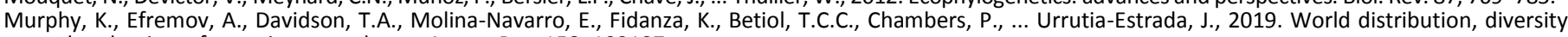
and endemism of aquatic macrophytes. Aquat. Bot. 158, 103127.

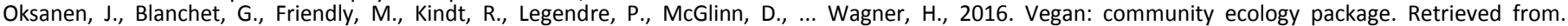
http://CRAN.R-project. org/package=vegan.

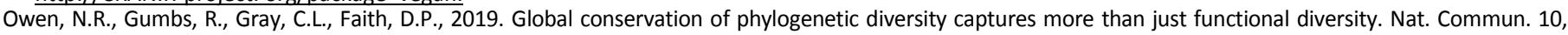
859.

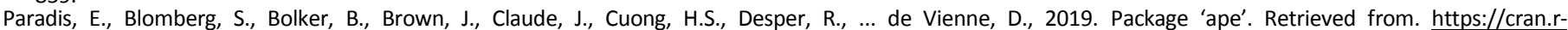
project.org/web/packages/ape/index.html.

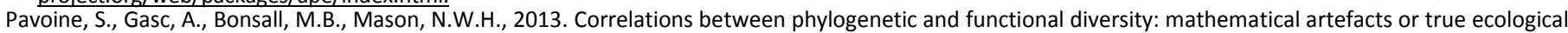
and evolutionary processes? J. Veg. Sci. 24, 781-793.

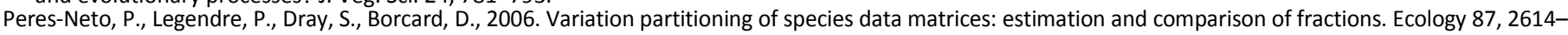
2625.

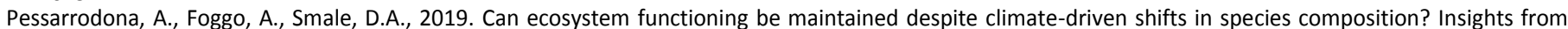
novel marine forests. J. Ecol. 107, 91-104.

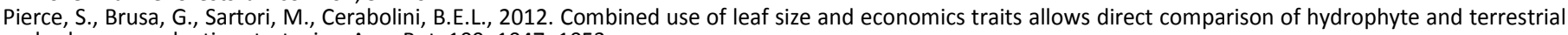
herbaceous adaptive strategies. Ann. Bot. 109, 1047-1053.

Poff, N.L., 1997. Landscape filters and species traits: towards mechanistic understanding and prediction in stream ecology. J. N. Am. Benthol. Soc. 61, 391-409. 
R Development Core Team, 2018. R: A Language and Environment for Statistical Computing. R Foundation for Statistical Computing, Vienna, Austria. Raup, D., Crick, R.E., 1979. Measurement of faunal similarity in paleontology. J. Paleontol. 53, $1213-1227$.

Revell, L., 2019. Package 'phytools'. Retrieved from. https://cran.r-project.org/web/packages/phytools/index.html.

Soininen, J., Lennon, J.J., Hillebrand, J., 2007. A multivariate analysis of beta diversity across organisms and environments. Ecology 88, 2830-2838.

Stevens, G.C., 1989. The latitudinal gradients in geographical range: how so many species co-exist in the tropics. Am. Nat. 133, 240-256.

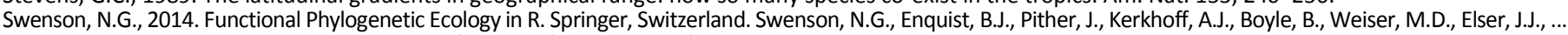
Nolting, K.M., 2012. The biogeography and filtering of woody plant functional diver-

sity in North and South America. Glob. Ecol. Biogeogr. 21, 798-808.

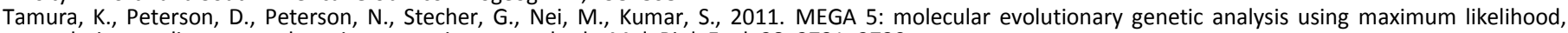
evolutionary distance, and maximum parsimony methods. Mol. Biol. Evol. 28, 2731-2739.

Whittaker, R.H., 1960. Vegetation of the Siskiyou Mountains, Oregon and California. Ecol. Monogr. 30, $280-338$.

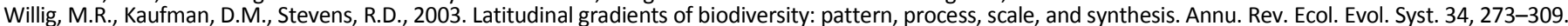

Wood, S.N., 2018. Package 'mgcv'. Retrieved from. https://cran.r-project.org/web/packages

/mgcv/index.html. 